\title{
Propuesta de modelo ODEAT para el diseño de intervenciones didácticas con integración de las TIC
}

\section{ODEAT Model for design of lesson planning with ICT integration}

\author{
* Hugo Tapia Silva, ${ }^{* *}$ Rodrigo Fernando Castillo Robledo, \\ *** Alexis Sarzosa Veliz \& **** Rodrigo López
}

Tapia, H., Castillo, R.F., Sarzosa, A., \& López, R. (2020). Propuesta de modelo ODEAT para el diseño de intervenciones didácticas con integración de las TIC. Revista Convergencia Educativa, 8, diciembre, 45-64.

DOI: http://doi.org/10.29035/rce.8.45

[Recibido: 10 julio, 2020 / Aceptado: 25 noviembre, 2020]

\begin{abstract}
RESUMEN
El presente estudio propone un modelo para guiar y facilitar la integración de las TIC en las prácticas pedagógicas, considerando las dificultades que supone la integración tecnológica en el contexto escolar, y, la revisión teórica de diversos autores, utilizando las siguientes propuestas -Grounded Technology Integration, Tipos de Actividades y Diseño Inverso-, las que permiten estructurar un proceso de diseño o planificación de clases, dando origen teóricamente al Modelo ODEAT. El modelo propuesto considera las etapas de: identificación del objetivo (0), la toma de decisiones pedagógicas (D), la definición de el o los indicadores de logro (E), la selección o creación de una actividad de aprendizaje (A), y la selección de la tecnología adecuada(T). Finalmente, se comentan las posibilidades y estrategias de evaluación del modelo.
\end{abstract}

Palabras clave: planificación de clases, integración de las TIC, profesores, didáctica, tecnología.

\footnotetext{
* Universidad de La Serena, La Serena, Chile. ORCID ID: https://orcid.org/0000-0002-2800-034X

** Universidad de La Serena, La Serena, Chile. ORCID ID: https://orcid.org/0000-0002-6872-3382

*** Liceo Bicentenario Colegio Antonio Varas, Vicuña, Chile. ORCID ID: https://orcid.org/0000-0001-6550-9930

**** Licenciado en Educación. Antofagasta, Chile. ORCID ID: https://orcid.org/0000-0001-5865-4240
} 
Tapia, H., Castillo, R.F., Sarzosa, A., \& López, R. (2020). Propuesta de modelo ODEAT para el diseño de intervenciones didácticas con integración de las TIC. Revista Convergencia Educativa, 8, diciembre, 45-64. DOI: http://doi.org/10.29035/rce.8.45

\section{ABSTRACT}

The present study proposes a model to guide and ease the integration of ICT on the pedagogical work, where it is considering the associate difficulties at the technological integration at the scholar context. Also, there is a theorical revision of various authors, where it is using the next proposal: Grounded Technology Integration, Types of Activities and Reverse Design. These proposals allow to structure a design or class planning process, where the ODEAT Model begins. The proposed model considers the stages of: identification of the objective (0), pedagogical decision-making (D), definition of the achievement indicator (s) (E), selection or creation of a learning activity (A), and the selection of the appropriate technology (T). Finally, the possibilities and strategies for evaluating the model are discussed.

Key words: Lesson plans, Technology Uses in Education, Teachers, Pedagogy, ICT.

\section{Introducción}

Los procesos de aprendizaje plantean una serie de desafíos a la integración de las Tecnologías de la Información y las Comunicaciones (en adelante, TIC). En primera instancia, es necesario resolver aspectos referentes al diseño de los procesos de aprendizaje (clases, unidades didácticas o procesos de mayor extensión), demandando en las y los profesores un nivel de conocimientos en ámbitos disciplinares, didácticos y tecnológicos. El nivel de estos conocimientos, independiente de la extensión del proceso, será un aspecto influyente en el diseño e integración resultante.

El diseño de intervenciones didácticas se ha identificado como uno de los conocimientos que debe tener el profesorado. Este conocimiento, desde la perspectiva de Lee Shulman, se encuentra relacionado con la etapa de transformación en el modelo de razonamiento y acción pedagógico (Shulman, 2005). Esta etapa considera procesos de preparación, representación, selección y adaptación de los conocimientos, los que son llevados a cabo por el profesorado con el fin de lograr los aprendizajes en sus estudiantes.

Sobre el diseño de procesos de aprendizaje integrando las TIC, nos planteamos las siguientes interrogantes: ¿Es posible identificar o diseñar una propuesta que podría facilitar este proceso al profesorado?, ¿qué posibilidades aportan estas orientaciones a las profesoras y profesores con conocimientos iniciales de tecnología educativa al momento de integrar las TIC en sus prácticas? Para responder estas preguntas, el siguiente ensayo expondrá el proceso de definición y construcción de una propuesta de modelo para el diseño de intervenciones didácticas con integración de las TIC en contextos educativos escolares, basada en la realidad del sistema educativo chileno.

\section{Metodología}

Para desarrollar el ensayo se ha utilizado una metodología basada en la revisión de políticas públicas y orientaciones que rigen la educación chilena, particularmente las que tratan la integración de tecnologías en las prácticas educativas. El criterio de selección de los documentos se basó en la pertinencia a la temática 
Tapia, H., Castillo, R.F., Sarzosa, A., \& López, R. (2020). Propuesta de modelo ODEAT para el diseño de intervenciones didácticas con integración de las TIC. Revista Convergencia Educativa, 8, diciembre, 45-64. DOI: http://doi.org/10.29035/rce.8.45

del ensayo "diseño de situaciones evaluativas integrando las TIC", con especial énfasis en las prácticas docentes asociadas a la integración.

Considerando lo anterior, se plantea una revisión de documentos oficiales para evidenciar las dificultades y retos de la integración de las TIC en las prácticas educativas de las y los profesores, considerando factores de índole personal y profesional en cuanto a utilización y diseño de situaciones didácticas.

En un tercer apartado, se fundamenta a partir de la revisión y descripción de propuestas de diseño didáctico con integración de tecnologías, planteadas por diversos autores, utilizando criterios como la relevancia temática, calidad de la información, originalidad y el aporte al conocimiento en el diseño de secuencias didácticas. Además, se describe cada una de las propuestas y sus pasos.

Finalmente, para la elaboración del modelo ODEAT, los criterios fueron la selección de las etapas más relevantes de cada propuesta descrita, considerando las necesidades de diseño planteadas por los documentos oficiales y las problemáticas al integrar las TIC en el ámbito educativo. Seguido de esto, se propone un modelo contextualizado a la realidad chilena, con secuencias debidamente detalladas que propician la reflexión y orientación en torno al proceso de diseño y planificación.

\section{Intervenciones didácticas con integración de TIC}

La integración de TIC en las prácticas pedagógicas se ve influida por factores personales y contextuales. Entre estos factores se pueden mencionar los conocimientos TIC, la percepción de autoeficacia, la disciplina formativa, la creencia sobre la importancia de las TIC, entre otros (Tapia, 2018). No obstante, existe escasa bibliografía sobre la relevancia del diseño de intervenciones didácticas para una buena integración de las TIC (Janssen \& Lazonder, 2015, Kwangsawad, 2016, Schmid, Brianza \& Petko, 2020), a pesar de la importancia que poseen estas intervenciones para una correcta integración de las TIC en los procesos formativos (Vásquez, 2011).

Un aspecto relevante que permite comprender esta situación es la dificultad para realizar la integración de la didáctica, el contenido y la tecnología. Esta dificultad de integración es especialmente interesante en los profesores nóveles (Janssen, Knoef \& Lazonder, 2019, Tapia \& Sobrino, 2019).

En el caso del contexto educativo chileno, existen orientaciones y referencias sobre el diseño de intervenciones didácticas a nivel general. En el caso de la integración de las TIC, existen limitadas orientaciones al respecto, pero en las políticas educativas podemos encontrar información relevante, tanto en las que son de carácter general como aquellas específicas relacionadas con la tecnología educativa. A continuación, repasaremos algunas orientaciones y resultados de estudios asociados al tema. 
Tapia, H., Castillo, R.F., Sarzosa, A., \& López, R. (2020). Propuesta de modelo ODEAT para el diseño de intervenciones didácticas con integración de las TIC. Revista Convergencia Educativa, 8, diciembre, 45-64. DOI: http://doi.org/10.29035/rce.8.45

\subsection{Marco para la Buena Enseñanza}

El Marco para la Buena Enseñanza (CPEIP, 2008), documento que presenta las bases de lo que se espera de una buena práctica pedagógica, establece la importancia del diseño de aprendizajes para el proceso formativo. En específico, en el Dominio de la Preparación de la Enseñanza, correspondiente al dominio A, se establecen los siguientes lineamientos:

- Los profesores y profesoras deben conocer la didáctica de las disciplinas que enseña (Criterio A.3), lo que en el modelo de Shulman equivale al Conocimiento didáctico del contenido. Es importante destacar que es necesario conocer y seleccionar distintos recursos de aprendizaje de acuerdo con los contenidos y los estudiantes.

- Se deben organizar los objetivos y contenidos de manera coherente con las bases curriculares (Criterio A.4). Esto implica un conocimiento claro del currículum escolar.

- Que las estrategias de evaluación desarrolladas sean coherentes con los objetivos de aprendizaje, la disciplina que enseña, las bases curriculares y que permita que todos los alumnos puedan demostrar lo que han aprendido (Criterio A.5).

En la actualización propuesta del MPBE del año 2018 (CPEIP y MINEDUC, 2018), en el Descriptor A.3.3 ya se hace una explicitación del uso de la tecnología, al mencionar que se espera que el profesorado incorpore "a la preparación de la enseñanza-aprendizaje recursos materiales y tecnológicos coherentes con la diversidad de sus estudiantes y los objetivos de aprendizaje de su disciplina" (p. 16). En el descriptor C.1.2, complementariamente, se señala que "para conseguir que sus explicaciones sean pertinentes a las características de sus estudiantes, utiliza (...) diversos recursos materiales y tecnológicos de apoyo a la explicación" (p. 36).

El MPBE, por tanto, entiende las tecnologías como un recurso que favorece el apoyo al abordaje de la diversidad, se vincula con el logro de los objetivos de aprendizaje y funciona como recurso para el proceso. Se requiere esperar para confirmar si estas propuestas se materializan en la próxima versión del MPBE.

\subsection{Marco de competencias y estándares TIC para la profesión docente}

A través de diversos marcos de competencias y estándares TIC, el Ministerio de Educación de Chile (en adelante, MINEDUC) y la Red Enlaces -institución del Ministerio de Educación responsables de la integración de las TIC en el contexto educativo chileno y que ahora ha pasado a denominarse Centro de Innovación- presentaron lineamientos sobre los conocimientos y habilidades básicas que deben poseer las y los integrantes del ámbito escolar al momento de integrar las TIC. En este contexto, se propuso una serie de documentos que pertenecen a la colección Marcos de competencias tecnológicas para el Sistema Escolar, 
Tapia, H., Castillo, R.F., Sarzosa, A., \& López, R. (2020). Propuesta de modelo ODEAT para el diseño de intervenciones didácticas con integración de las TIC. Revista Convergencia Educativa, 8, diciembre, 45-64. DOI: http://doi.org/10.29035/rce.8.45

cuyas versiones son Competencias y estándares TIC para directores, para la Profesión Docente, para Jefes de Unidad Técnico Pedagógico, para Orientadores y para Coordinadores de las Bibliotecas Escolares CRA.

En el caso del profesorado, se definieron cinco dimensiones de competencias a desarrollar durante el ejercicio de su profesión docente (MINEDUC y ENLACES, 2011): Pedagógica, Gestión, Técnica, Desarrollo y responsabilidad profesional, y Social ética y legal. Si bien cada una de estas dimensiones aborda un aspecto de la práctica educativa del profesorado, en esta ocasión destacaremos la dimensión pedagógica. Cada dimensión contempla una serie de competencias, y cada competencia dispone de una serie de criterios que describen lo que debería demostrar un profesor o profesora competente.

En específico, en la dimensión pedagógica se establecen tres competencias. En la primera competencia se establece que el profesorado debe "Integrar TIC en la planificación de ambientes y experiencias de aprendizaje de los sectores curriculares para agregar valor al aprendizaje y al desarrollo integral de los estudiantes" (p. 29). Para esa competencia se establecen 4 criterios, desde el 1.1.1 al 1.1.4, que describen las capacidades que deben poseer profesoras y profesores en su práctica pedagógica. Por tanto, un profesorado competente es aquel que:

1.1.1. Planifica ambientes y experiencias de aprendizaje utilizando resultados de estudios, buenas prácticas o estrategias probadas respecto del uso de TIC.

1.1.2. Diagnostica el contexto para planificar el uso de TIC en el diseño de actividades de aprendizaje y de acuerdo a los recursos disponibles.

1.1.3. Selecciona o adapta recursos digitales para potenciar el aprendizaje de los estudiantes de acuerdo a las oportunidades, normativas, materiales y humanas del contexto de desempeño.

1.1.4. Diseña estrategias de evaluación utilizando recursos digitales pertinentes a los aprendizajes esperados.

La revisión de estos indicadores sugiere una serie de inquietudes sobre el trabajo que deben realizar las y los docentes previo a la implementación de una clase: Planificar, diagnosticar, seleccionar o adaptar y evaluar el logro de aprendizajes. Resulta interesante que se considere la planificación como un momento separado de la toma de decisiones en torno a la evaluación, lo que sugiere dos procesos aislados, el proceso evaluativo por un lado y planificación de la intervención didáctica por otro.

Un estudio reciente de Arancibia, Cosimo \& Casanova (2018) señalan que el profesorado reconoce un desarrollo en la habilidad de integrar las TIC en las planificaciones de los procesos de aprendizaje, y a la vez, la optimización del orden de las planificaciones, en el marco de la normativa actual. 
Tapia, H., Castillo, R.F., Sarzosa, A., \& López, R. (2020). Propuesta de modelo ODEAT para el diseño de intervenciones didácticas con integración de las TIC. Revista Convergencia Educativa, 8, diciembre, 45-64. DOI: http://doi.org/10.29035/rce.8.45

\subsection{Algunos datos de uso: el Censo TIC}

En el contexto chileno, el uso de las tecnologías en las escuelas presenta un alza permanente. Los datos del Censo TIC (Instituto de Informática Educativa y Adimark GFK, 2013) dan cuenta de que un 97\% de los profesores dice utilizar el computador semanalmente; también se destaca un uso del laboratorio de computación que es transversal, aunque con una frecuencia relativa según la asignatura. También se señala que un $90 \%$ del profesorado de contextos urbanos cuenta con acceso a computadores y sobre el 50\% con acceso a Internet. Estos datos son levemente menores en establecimientos de contexto rural. En el Ámbito de Uso, la Planificación pedagógica con uso de TIC se menciona como aspecto importante de las acciones de apoyo a la enseñanza.

En el contexto de la educación rural, los datos señalan que el porcentaje de directores de establecimientos que implementa incentivos para el uso de las TIC es de un $23 \%$, un tercio de lo que ocurre en el contexto urbano. Si se consideran también los datos sobre necesidades de capacitación, se observa que las principales preocupaciones que manifestaron profesores y profesoras de contexto rural hacen referencia a la integración de las TIC.

Los datos mencionados nos permiten identificar una preocupación por parte de las direcciones de los establecimientos educativos para generar condiciones que favorezcan la integración de las TIC en la planificación de intervenciones didácticas.

\section{Dificultades para la integración de las TIC}

Como hemos mencionado, la integración de las TIC por parte del profesorado en los procesos de aprendizaje depende de variables tanto personales como de contexto. Entre ellas, la revisión teórica menciona como algunas de las relevantes: a) nivel de conocimientos y habilidades TIC que posee un docente, pues ellos definirán las herramientas digitales que están en condiciones de utilizar en sus prácticas pedagógicas y educativas (Avidov \& Eshet, 2011, Drossel, Eickelmann \& Gerick, 2017, Dela Rosa, 2016); b) las expectativas de esfuerzo (Birch \& Irvine, 2009), es decir, el esfuerzo (tiempo, dinero, otros) que un profesor considera que le costará integrar tecnologías en sus clases; y c) percepción de autoeficacia, que hace referencia a la autopercepción sobre la capacidad de llevar a cabo la integración de las TIC en sus clases (Sang, Valcke, van Braak \& Tondeur, 2010, Hammond, Reynolds \& Ingram, 2011).

Estos factores, asociados a la integración de las TIC del profesorado en sus prácticas pedagógicas, definen a la vez un actuar personal en cuanto al uso de las tecnologías. Por tanto, las propuestas de integración deben considerar que existen diferentes tipos y frecuencias de uso por parte del profesorado al momento de utilizar las TIC, tanto en el ámbito personal como profesional. 
Tapia, H., Castillo, R.F., Sarzosa, A., \& López, R. (2020). Propuesta de modelo ODEAT para el diseño de intervenciones didácticas con integración de las TIC. Revista Convergencia Educativa, 8, diciembre, 45-64. DOI: http://doi.org/10.29035/rce.8.45

\section{Diseño de intervenciones didácticas y TIC}

Como se ha mencionado, la planificación de clase o de intervenciones didácticas surge como un aspecto de gran importancia en el proceso de integración de las TIC en el aula de clases y las prácticas educativas. No obstante, la evidencia muestra una baja preocupación en la investigación sobre el proceso de diseño integrando las TIC y cómo lo lleva a cabo el profesorado (Koh \& Chai, 2016). En este contexto, consideramos relevante investigar y proponer métodos para los procesos de diseño de intervenciones didácticas que consideren las tecnologías, a fin de alcanzar una integración de las TIC que favorezca el logro de aprendizajes por parte de los estudiantes.

El proceso de diseño de una situación de aprendizaje es un acto reflexivo y práctico al mismo tiempo (Hidson, 2018), que se caracteriza, además, tal como señalan Gvirtz \& Palamidessi (2011), por su carácter proyectivo. Estas tres características del proceso de diseño suponen un desafío a la integración de las TIC, pues el diseño de una situación de aprendizaje es un paso relevante para la integración de las TIC (Soalablai, 2020). Se espera que un diseño debiera disponer de un marco de referencia teórico que fundamente las decisiones. En el contexto actual, tres modelos teóricos actuales resultan relevantes: TIM, SAMR y TPACK (Kolb, 2017). En especial, el modelo TPACK se ha convertido en un referente importante para la presencia del uso de las TIC en contextos educativos. Este modelo supone la interrelación entre los conocimientos pedagógicos, didácticos y tecnológicos (Tapia, 2017), de forma que la comprensión profunda de cada uno de estos conocimientos y sus interrelaciones favorece el proceso de integración y, por consecuencia, en el diseño de las situaciones de aprendizaje.

\subsection{Grounded technology integration}

Uno de los acercamientos al diseño de clases integrando las TIC, y que se encuentra vinculado al modelo TPACK, es la propuesta de Harris y Hofer (Harris \& Hofer, 2009b, Harris \& Hofer, 2009a, Harris, Hofer, Blanchard, Grandgenett, Schmidt, Van Olphen \& Young, 2010), denominada Grounded technology integration (o GTI). Los autores proponen un modelo de diseño de clases que considera cinco etapas que debe considerar un docente. La siguiente tabla presenta y describe cada una de las cinco etapas del modelo: 
Tapia, H., Castillo, R.F., Sarzosa, A., \& López, R. (2020). Propuesta de modelo ODEAT para el diseño de intervenciones didácticas con integración de las TIC. Revista Convergencia Educativa, 8, diciembre, 45-64. DOI: http://doi.org/10.29035/rce.8.45

Tabla 1

Etapas y descripción del modelo Grounded Technology Integration.

\begin{tabular}{ll}
\hline Etapa & Descripción \\
\hline Identificar objetivos & $\begin{array}{l}\text { Selección o creación del objetivo de aprendizaje correspondiente al } \\
\text { proceso de aprendizaje. }\end{array}$ \\
& Define las características básicas de su propuesta a partir de ocho \\
& cuestiones o aspectos, incluyendo, por ejemplo, el tiempo disponible, la \\
Tomar decisiones pedagógicas & $\begin{array}{l}\text { agrupación del grupo curso, el nivel cognitivo que se desea alcanzar, } \\
\text { entre otros. }\end{array}$ \\
\hline Seleccionar actividad & Selección o diseño de la actividad de aprendizaje en que participarán \\
& los estudiantes. \\
Seleccionar estrategias de evaluación & $\begin{array}{l}\text { A partir de la actividad definida, se selecciona cómo se evaluarán los } \\
\text { aprendizajes que se deberían lograr al finalizar el proceso. }\end{array}$ \\
\hline Seleccionar herramientas y recursos & Para realizar la actividad y la evaluación del logro de aprendizaje, en \\
& esta última etapa se define la herramienta tecnológica a emplear. \\
\hline
\end{tabular}

Fuente: elaboración propia.

Dos aspectos definen la propuesta de los autores: en primer lugar, sugieren que las estrategias de integración de las TIC deben incluirse en la forma de planificación docente, en vez de planificar intentando coincidir con las tecnologías educativas. En segundo lugar, adoptan un enfoque basado en tipos de actividades que se integran al proceso de diseño de la intervención didáctica.

La Figura 1 esquematiza los momentos de este modelo:

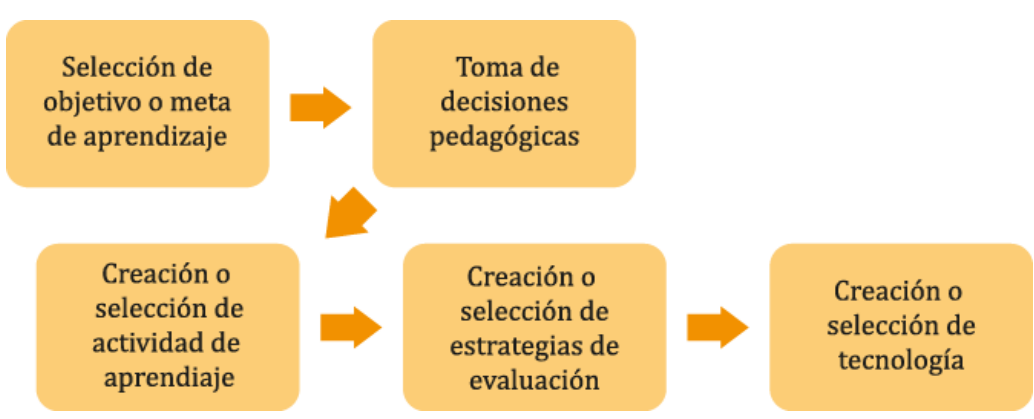

Figura 1. Etapas del modelo Grounded Technology Integration. Fuente: elaboración propia. 
Tapia, H., Castillo, R.F., Sarzosa, A., \& López, R. (2020). Propuesta de modelo ODEAT para el diseño de intervenciones didácticas con integración de las TIC. Revista Convergencia Educativa, 8, diciembre, 45-64. DOI: http://doi.org/10.29035/rce.8.45

\subsection{Tipos de actividades de aprendizaje}

Las actividades de aprendizaje que se pueden integrar en una clase pueden ir desde aquellas que podemos considerar más simples (por ej.: leer una página web, ver una foto, escuchar una canción) a otras actividades mucho más complejas (por ej.: crear una revista electrónica, fotografiar monumentos o interpretar una canción). Bajo esta premisa, Harris y Hofer han elaborado una serie de taxonomías de actividades para distintas disciplinas en las que se listan tipos de actividades, organizadas de acuerdo con enfoques teóricos o modelos formativos adecuados a las características de la disciplina (Harris, 2011, Harris \& Hofer, 2011). Cada una de estas taxonomías otorga un marco práctico para la integración de las tecnologías, pues relaciona una actividad de aprendizaje a una tecnología adecuada.

La combinación de actividades puede generar estrategias más complejas de enseñanza. Harris, Mishra \& Koehler (2009) señalan que cuando aumenta la cantidad de actividades que son combinadas, se puede observar la complejidad que enfrentan los futuros profesores o la estructura que adopta la formación.

En las tres siguientes tablas se presentan los tipos de actividades que han definido los autores, distribuidas según nueve asignaturas o grupos de asignaturas.

Tabla 2

Taxonomía de Tipos de actividades de aprendizaje según disciplina, parte 1.

\begin{tabular}{lll}
\hline Lenguaje & Lengua extranjera & Lengua y literatura nivel secundario \\
\hline Previas a la lectura & De escucha & Tipos de actividades del proceso de lectura \\
Durante la lectura & De habla & Tipos de actividades del proceso de escritura \\
Posteriores a la lectura & De escritura & Tipos de actividades enfocadas en el lenguaje \\
De vocabulario & De lectura & Tipos de actividades de lengua oral/desempeño \\
Comprensión lectora & De visionado & Tipos de actividades de escucha/visionado \\
De fluidez & & \\
Previas a la escritura & & \\
Durante la escritura & \\
Posterior a la escritura & \\
De convenciones de la escritura & \\
De escritura de género & \\
\hline
\end{tabular}

Fuente: elaboración propia basado en Harris (2011) y Harris \& Hofer (2011). 
Tapia, H., Castillo, R.F., Sarzosa, A., \& López, R. (2020). Propuesta de modelo ODEAT para el diseño de intervenciones didácticas con integración de las TIC. Revista Convergencia Educativa, 8, diciembre, 45-64. DOI: http://doi.org/10.29035/rce.8.45

Tabla 3

Taxonomía de Tipos de actividades de aprendizaje según disciplina, parte 2.

\begin{tabular}{lll}
\hline Ciencias sociales & Música & Artes visuales \\
\hline $\begin{array}{ll}\text { Construcción de conocimiento } \\
\text { Expresión de conocimiento }\end{array}$ & De improvisación & $\begin{array}{l}\text { Construir conocimiento } \\
\text { /Conceptualizar" }\end{array}$ \\
$\begin{array}{l}\text { Expresión convergente de } \\
\text { conocimientos }\end{array}$ & De canto & Aplicar \\
$\begin{array}{l}\text { Expresión divergente de } \\
\text { conocimientos }\end{array}$ & De ejecución de instrumentos & Responder \\
$\begin{array}{l}\text { Expresión visual divergente de } \\
\text { conocimientos }\end{array}$ & De lectura y notación musical \\
$\begin{array}{l}\text { Expresión divergente de } \\
\text { conocimientos conceptuales }\end{array}$ & De escucha y descripción & Describir \\
$\begin{array}{l}\text { Expresión divergente de } \\
\text { conocimientos orientadas a la } \\
\text { elaboración de productos }\end{array}$ & De análisis musical & Analizar/Interpretar \\
$\begin{array}{l}\text { Participativas de expresión } \\
\text { divergente de conocimientos }\end{array}$ & De evaluación musical & \\
& $\begin{array}{l}\text { De relación entre música, otras } \\
\text { artes y disciplinas no artísticas }\end{array}$ & \\
\hline
\end{tabular}

Fuente: elaboración propia basado en Harris (2011) y Harris \& Hofer (2011).

Tabla 4

Taxonomía de Tipos de actividades de aprendizaje según disciplina, parte 3.

\begin{tabular}{lll}
\hline Matemáticas & Ciencias & Educación Física \\
\hline Para considerar & $\begin{array}{l}\text { Construcción de conocimientos } \\
\text { conceptuales } \\
\text { Construcción de conocimientos } \\
\text { procedimentales }\end{array}$ & $\begin{array}{l}\text { Aptitud física: desarrollo del } \\
\text { conocimiento }\end{array}$ \\
Para practicar & Expresión de conocimientos & $\begin{array}{l}\text { Aptitud física: aplicación del } \\
\text { conocimiento }\end{array}$ \\
Para interpretar & Psicomotriz: práctica \\
Para producir & $\begin{array}{l}\text { Psicomotriz: aplicación } \\
\text { Para aplicar }\end{array}$ & $\begin{array}{l}\text { Desarrollo de la habilidad motriz: } \\
\text { desarrollo del conocimiento }\end{array}$ \\
Para evaluar & $\begin{array}{l}\text { Desarrollo de la habilidad motriz: } \\
\text { aplicación del conocimiento }\end{array}$ \\
\hline Para crear & & \\
\hline
\end{tabular}

Fuente: elaboración propia basado en Harris (2011) y Harris \& Hofer (2011). 
Tapia, H., Castillo, R.F., Sarzosa, A., \& López, R. (2020). Propuesta de modelo ODEAT para el diseño de intervenciones didácticas con integración de las TIC. Revista Convergencia Educativa, 8, diciembre, 45-64. DOI: http://doi.org/10.29035/rce.8.45

Los tipos de actividades se organizan incluyendo cinco elementos. En primer lugar, se identifica la disciplina formativa. A continuación, se revisa el documento y se identifica el tipo de actividad que nos interesa que los estudiantes realicen. El tercer momento implica seleccionar la actividad específica considerada preliminarmente adecuada para que realicen los estudiantes de acuerdo con el objetivo del proceso; este paso se une al siguiente, en el cual debemos leer la descripción de la actividad para evaluar su pertinencia. Finalmente, si la actividad nos parece la adecuada, identificamos en la propuesta de Tipos de actividades cuál es la herramienta digital más adecuada para mi propuesta. La siguiente figura esquematiza el proceso de análisis para la integración de las TIC:

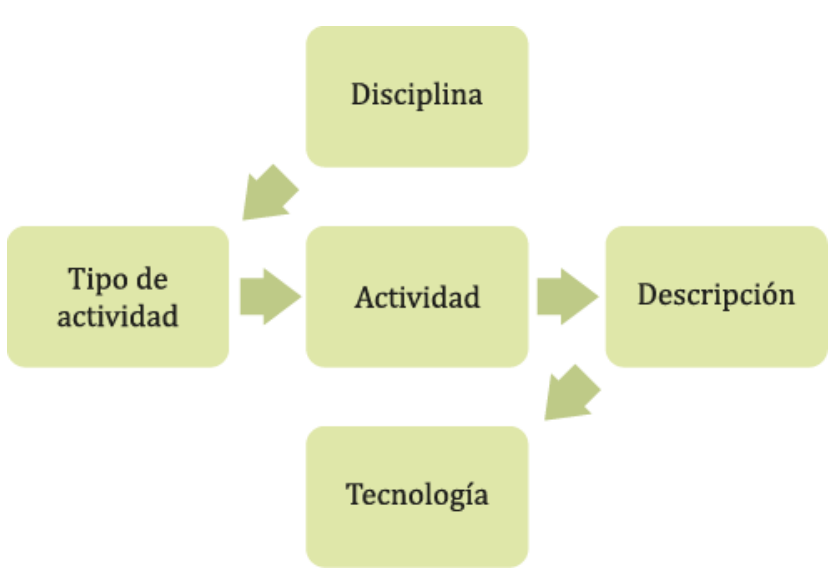

Figura 2. Componentes del modelo de Tipos de actividades de aprendizaje. Fuente: elaboración propia.

\subsection{Diseño inverso}

El modelo de Diseño inverso propone un enfoque no habitual para el diseño de aprendizajes. Su propuesta se basa en que la planificación de una actividad formativa debe poseer coherencia interna, frente a modelos que entienden que algunos aspectos son independientes. Un ejemplo de ello se observa en el Marco de competencias para la profesión docente y la separación que se realiza entre diseño de aprendizajes y evaluación.

En general, los autores adheridos a este modelo han establecido que el proceso de diseño de aprendizajes debe considerar tres momentos, si bien sus propuestas difieren levemente en la identificación de algunos aspectos. Para algunos autores, son tres momentos a) identificación de resultados esperados del proceso; b) elaboración de evidencias; y c) actividades de aprendizaje (Said, 2013, Wiggins \& McTighe, 2005). 
Tapia, H., Castillo, R.F., Sarzosa, A., \& López, R. (2020). Propuesta de modelo ODEAT para el diseño de intervenciones didácticas con integración de las TIC. Revista Convergencia Educativa, 8, diciembre, 45-64. DOI: http://doi.org/10.29035/rce.8.45

Por otra parte, García \& Cabero (2017) identifican los tres pasos del modelo como a) resultados esperados, b) evidencias y c) planificación de contenidos. Para Olivares, Angulo, Prieto \& Torres (2018, p. 30) los pasos que componen el modelo de diseño inverso corresponden a "a) identificar los resultados esperados, b) seleccionar las evidencias de evaluación y c) elaborar las micro experiencias de aprendizaje".

Como se observa, en términos concretos, la propuesta de diseño inverso supone que se debe centrar el diseño de las intervenciones didácticas en responder al logro de los resultados que se espera del proceso de aprendizaje. Esto implica que la etapa de diseño de la evaluación (definición de indicadores de logro y/o estrategia de evaluación) se realiza una vez que la meta de aprendizaje se ha establecido claramente y actúa como referencia del proceso posterior. Una vez realizado el paso anterior, corresponde al momento de establecer los contenidos relacionados y las actividades de aprendizaje que permitirán que los estudiantes puedan dar evidencia del logro esperado. La siguiente figura presenta los momentos del diseño inverso en sus dos variantes.

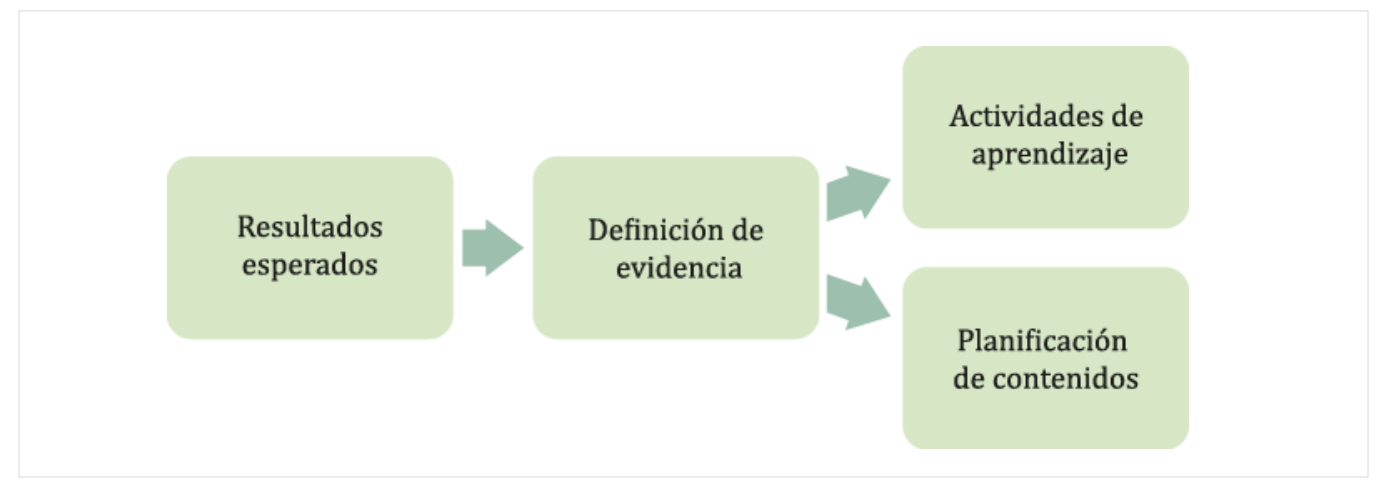

Figura 3. Etapas del modelo de Diseño inverso. Fuente: elaboración propia.

\section{Modelo ODEAT}

A partir de las tres propuestas anteriores, proponemos un modelo para el diseño de intervenciones didácticas que integren las TIC para ser utilizado en el contexto escolar.

La propuesta que realizamos surge de la identificación de los aportes de cada uno de estos elementos teóricos al proceso de diseño de clases y de intervenciones didácticas, reconociendo sus semejanzas y diferencias, y a partir de la consideración de estos aspectos, construir un modelo nuevo que mantenga los elementos fundantes de cada uno de ellos.

A partir de Grounded technology integration, destacamos las cinco etapas básicas que proponen, en cuanto permiten organizar el proceso de diseño de intervenciones didácticas. La estructura general que 
Tapia, H., Castillo, R.F., Sarzosa, A., \& López, R. (2020). Propuesta de modelo ODEAT para el diseño de intervenciones didácticas con integración de las TIC. Revista Convergencia Educativa, 8, diciembre, 45-64. DOI: http://doi.org/10.29035/rce.8.45

presentan destaca por considerar como último paso la selección de la herramienta TIC que mejor responda a las decisiones tomadas en los cuatro primeros momentos.

Tras analizar los Tipos de aprendizaje, destacamos su rol orientador para la identificación, selección e integración de herramientas TIC de acuerdo con las necesidades expresadas por el profesorado relacionadas con los objetivos o metas de aprendizaje. Si lo relacionamos con el modelo Grounded technology integration, su uso se sitúa en el momento 3 , y responde a la necesidad de identificar herramientas específicas relacionadas con tipos de actividades concretas.

El modelo Inverso orienta la definición final de la estructura del modelo propuesto. A partir de este modelo, reconocemos la importancia de situar el componente evaluación en un momento previo a la finalización del diseño de la clase. En nuestro caso, lo situamos entre las decisiones pedagógicas y la creación o selección de la actividad de aprendizaje.

Finalmente, redefinimos y construimos un modelo que considera 5 momentos. A continuación, presentamos la descripción de cada uno de los momentos de nuestra propuesta de modelo:

1. Selección de Objetivo. La primera etapa de nuestro proceso implica la selección o redacción del Objetivo de Aprendizaje (meta, objetivo, propósito, etc) que se abordará en el proceso de enseñanzaaprendizaje. Responde, por tanto, en la definición inicial de todo proceso formativo.

2. Toma de Decisiones pedagógicas. Consiste en la definición didáctica en torno a ocho cuestiones que se encuentran relacionadas con los fundamentos y la construcción del ambiente de aprendizaje. Estas decisiones hacen referencia a 8 cuestiones concretas, que responden a las siguientes preguntas:

a. ¿En quién radica la centralidad del proceso, en el profesor o profesora o en el estudiante?

b. ¿Qué tipo de aprendizaje (enfoque) caracterizará el proceso?

c. ¿Cuál es la importancia que poseen los conocimientos previos en el proceso formativo?

d. ¿Qué nivel de profundidad se alcanzará en los conocimientos conceptuales, procedimentales o actitudinales?

e. ¿Cuál es el grado de estructuración del proceso?

f. ¿Cómo se distribuyen los estudiantes durante el proceso?

g. ¿Cuál es la cantidad de recursos tecnológicos necesarios/disponibles para el proceso?

3. Evaluación. En un tercer momento corresponde a la realización de definiciones relativas a la evaluación del desempeño de los estudiantes. Implica tomar decisiones en torno al indicador de aprendizaje y las estrategias de evaluación que se considerarán sobre él. 
Tapia, H., Castillo, R.F., Sarzosa, A., \& López, R. (2020). Propuesta de modelo ODEAT para el diseño de intervenciones didácticas con integración de las TIC. Revista Convergencia Educativa, 8, diciembre, 45-64. DOI: http://doi.org/10.29035/rce.8.45

4. Selección o diseño de Actividad de aprendizaje. Una vez establecidos el indicador y/o la estrategia de evaluación, el siguiente paso consiste en la identificación de la actividad asociada a estos dos elementos. Para ello, se propone el empleo de la Taxonomía de Tipos de Actividades de J. Harris y M. Hofer (Harris, 2011, Hofer \& Harris, 2011), la cual entrega una serie de actividades, clasificadas según complejidad u objetivo.

5. Creación o selección de Herramienta TIC. Empleando la Taxonomía propuesta o los conocimientos con que cuenta el o la docente, se selecciona (o crea) la herramienta TIC más adecuada para el logro del indicador previamente definido y que sea pertinente a la actividad de aprendizaje propuesta.

La propuesta del modelo ODEAT -acrónimo de Objetivo, Decisiones, Evaluación, Actividad y Tecnologíaimplica la centralidad de la evaluación y su proceso, situando como último espacio de decisión y diseño, la selección de la herramienta tecnológica pertinente. Este modelo propuesto, por tanto, reconoce los principales aportes de cada uno de los modelos analizados anteriormente. A partir de él, se propone que el profesorado genere un espacio de reflexión sobre sus intervenciones didácticas, al mismo tiempo que le otorgue orientaciones para el diseño de estas intervenciones.

A continuación, en la Figura 4, presentamos el esquema del Modelo ODEAT.

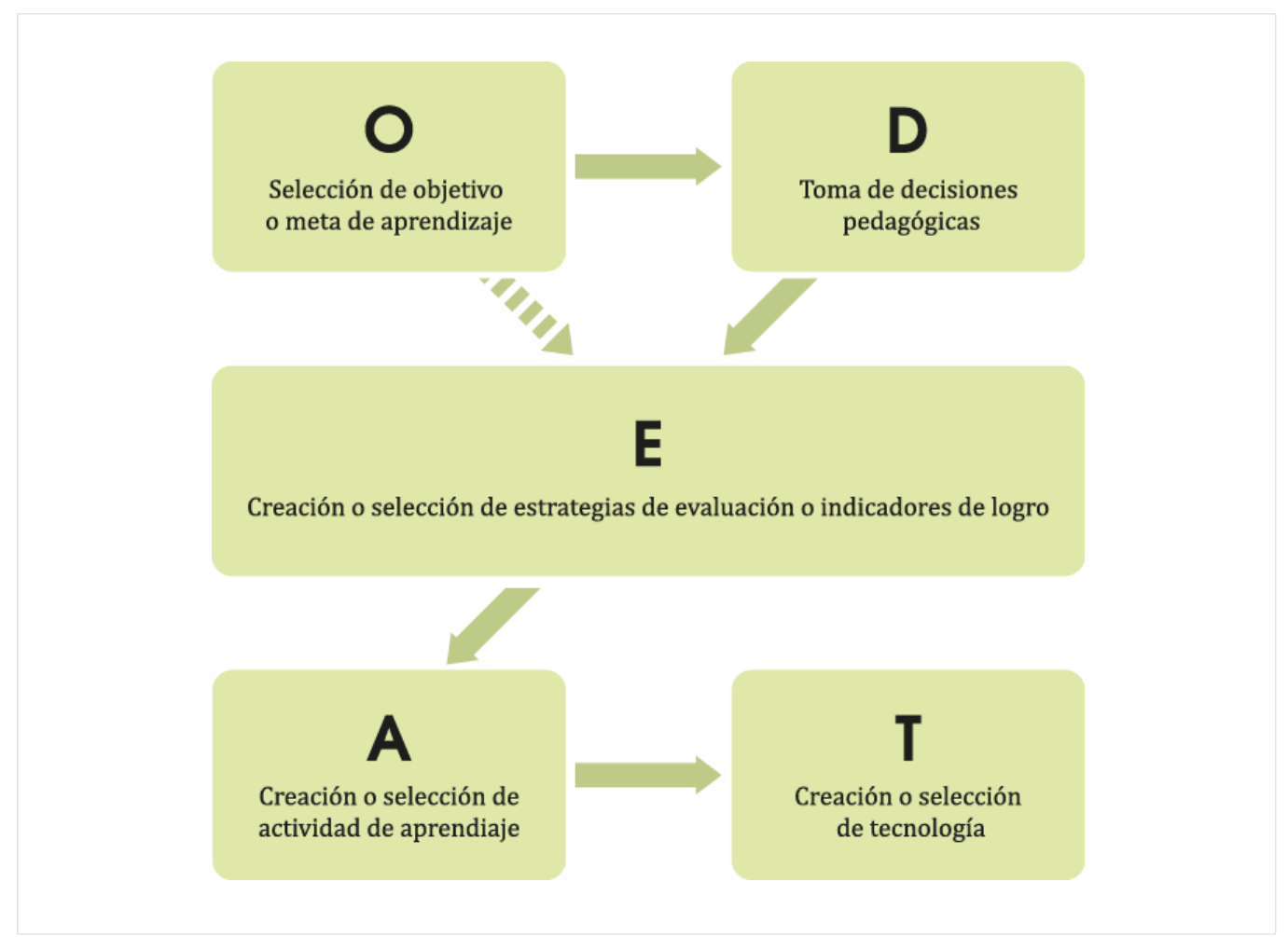

Figura 4. Modelo ODEAT. Fuente: elaboración propia. 
Tapia, H., Castillo, R.F., Sarzosa, A., \& López, R. (2020). Propuesta de modelo ODEAT para el diseño de intervenciones didácticas con integración de las TIC. Revista Convergencia Educativa, 8, diciembre, 45-64. DOI: http://doi.org/10.29035/rce.8.45

El punto de inicio del modelo es la selección del objetivo (0). El paso posterior corresponde a la toma de Decisiones pedagógicas (D); desde ese momento, se evidencia la centralidad del momenot de creación o selección de estrategias de evaluación o indicadores de logro (E). Como se obsera, desde (O) se dirige a (E) una flecha segmentada. Esta flecha segmentada indica la relación que debe existir entre ambos elementos. Una vez definido (E), se continúa en el creación o selección de la actividad de aprendizaje (A), para, finalmente, crear o seleccionar la herramienta pertinente al proceso (T).

\section{Discusión y conclusiones}

El modelo ODEAT se presenta como una oportunidad que podría suponer diferentes beneficios para el profesorado en el proceso de diseño de procesos de aprendizaje con integración de tecnologías.

Las tres bases del modelo -Grounded Technology Integration, Tipos de actividades de aprendizaje y Diseño inverso- permiten dotar de coherencia a un modelo de diseño de procesos de integración de tecnología en las prácticas pedagógicas. Su valor se encuentra en la resignificación del proceso posponiendo la selección de la herramienta tecnológica a un momento final de la planificación didáctica.

Se reconoce, a partir del modelo, lo importante que es considerar las características del proceso que se está diseñando al momento de tomar de decisiones, ya que esto provee una guía de diseño coherente con las necesidades formativas del contexto.

Desde la perspectiva del modelo de Shulman, consideramos que la estructura y elementos fundantes del modelo permiten orientar especialmente al profesorado en formación y al profesorado novel. Aumentar el número de representaciones que permiten a los profesores lograr la comprensión por parte de sus estudiantes es un aspecto relevante en la práctica pedagógica. En este sentido, creemos que puede ser útil emplear el modelo en la formación inicial de profesores, con el fin de generar un proceso reflexivo en torno al uso de las tecnologías.

Como tal, el modelo requiere ser evaluado empíricamente. Se propone, para tal efecto, realizar una investigación con profesores y profesoras del sistema escolar en que se evalúe su pertinencia para ser utilizado en sus prácticas pedagógicas para el diseño de intervenciones didácticas. A partir de una investigación de este tipo, y considerando las recomendaciones y críticas de los docentes que se encuentren empleando el modelo, se deben realizar las modificaciones que sean necesarias para mejorar el modelo al contexto específico. Esta evaluación debería desarrollarse a partir del modelo investigación-acción. 
Tapia, H., Castillo, R.F., Sarzosa, A., \& López, R. (2020). Propuesta de modelo ODEAT para el diseño de intervenciones didácticas con integración de las TIC. Revista Convergencia Educativa, 8, diciembre, 45-64. DOI: http://doi.org/10.29035/rce.8.45

Finalmente, se reconoce en el modelo la factibilidad de que pueda ser empleado para el diseño de clases que no impliquen, necesariamente, el uso de tecnología. Esto es posible, si consideramos que las primeras cuatro etapas del modelo (O-D-E-A) no realizan una referencia directa al uso de la tecnología para el aprendizaje, si no poseen un carácter general. Sería necesario, para ello, revisar qué aspectos requieran ser redefinidos de los primeros pasos del modelo o generar una versión que considere una referencia explícita a recursos.

\section{REFERENCIAS BIBLIOGRÁFICAS}

Adimark GFK -IIE. (2013). Censo de Informática Educativa 2012. Resultados principales. (Census Report ed.). Recuperado de https://paisdospuntocero.files.wordpress.com/2015/04/03censo_de_informatica_educativa3.pdf

Arancibia Herrera, M., Cosimo Fernández, D., \& Casanova Seguel, R. (2018). Percepción de los profesores sobre integración de TIC en las prácticas de enseñanza en relación a los marcos normativos para la profesión docente en Chile. Ensaio: Avaliação e Políticas Públicas em Educação, 26(98), 163-184. DOI: https://doi.org/10.1590/s0104-40362017002501119

Avidov Ungar, O., \& Eshet Alkalay, Y. (2011). Teachers in a World of Change: Teachers' Knowledge and Attitudes towards the Implementation of Innovative Technologies in Schools. Interdisciplinary Journal of E-Learning and Learning Objects, (7), 291-303. Recuperado de https://www.learntechlib.org/p/44745/

Birch, A., \& Irvine, V. (2009). Preservice teachers' acceptance of ICT integration in the classroom: applying the UTAUT model. Educational Media International, 46(4), 295-315. DOI: https://doi.org/10.1080/09523980903387506

CPEIP. (2008). Marco para la Buena Enseñanza. Santiago de Chile: Ministerio de Educación. Recuperado de https://www.cpeip.cl/wp-content/uploads/2019/01/Marco-buena-ensenanza.pdf

CPEIP. (2018). Marco para la Buena Enseñanza. Santiago de Chile: Ministerio de Educación. Recuperado de https://www.cpeip.cl/marco-buena-ensenanza/ 
Tapia, H., Castillo, R.F., Sarzosa, A., \& López, R. (2020). Propuesta de modelo ODEAT para el diseño de intervenciones didácticas con integración de las TIC. Revista Convergencia Educativa, 8, diciembre, 45-64. DOI: http://doi.org/10.29035/rce.8.45

Dela Rosa, J. (2016). Experiences, perceptions and attitudes on ICT integration: A case study among novice and experienced language teachers in the Philippines. International Journal of Education and Development using Information and Communication Technology, 12(3), 37-57. Recuperado de https://files.eric.ed.gov/fulltext/EJ1124823.pdf

Drossel, K., Eickelmann, B., \& Gerick, J. (2017). Predictors of teachers' use of ICT in school - the relevance of school characteristics, teachers' attitudes and teacher collaboration. Education and Information Technologies, (22), 551-573. DOI: https://doi.org/10.1007/s10639-016-9476-y

García Marcos, C. J., \& Cabero Almenara, J. (2017). El diseño instruccional inverso para un recurso educativo abierto en la Formación Profesional española: El caso de Web Apps Project. Education in the Knowledge Society, 18(2), 19-32. DOI: https://doi.org/10.14201/eks20171821932

Gvirtz, S., \& Palamidessi, M. (2011). El ABC de la tarea docente: currículum y enseñanza. Capital Federal: Aique. Recuperado de http://www.unter.org.ar/imagenes/10062.pdf

Hammond, M., Reynolds, L., \& Ingram, J. (2011). How and why do student teachers use ICT? Journal of Computer Assisted Learning, 27(3), 191-203. DOI: https://doi.org/10.1111/j.13652729.2010.00389.x

Harris, J. (2011). Activity Types Wiki. Recuperado de https://activitytypes.wm.edu/

Harris, J., \& Hofer, M. (2009a). Instructional planning activity types as vehicles for curriculum-based TPACK development. En C. D. Maddux, (Ed.), Research highlights in technology and teacher education (pp. 99-108). Chesapeake, VA: Society for information. Recuperado de https://www.academia.edu/1778414/Instructional_planning_activity_types_as_vehicles_for_curr iculum_based_TPACK_development

Harris, J., \& Hofer, M. J. (2009b). Grounded tech integration: An effective approach based on content, pedagogy, and teacher planning. Learning \& Leading with Technology, 37(2), 22-25. Recuperado de https://scholarworks.wm.edu/articles/28/

Harris, J., Hofer, M., Blanchard, M., Grandgenett, N., Schmidt, D., Van Olphen, M., \& Young, C. (2010). "Grounded" technology integration: Instructional planning using curriculum-based activity type taxonomies. Journal of Technology and Teacher Education, 18(4), 573-605. Recuperado de https://digitalcommons.unomaha.edu/cgi/viewcontent.cgi?article=1040\&context=tedfacpub

Harris, J., Mishra, P., \& Koehler, M. J. (2009). Teachers' technological pedagogical content knowledge and learning activity types: Curriculum-based technology integration reframed. Journal of Research on $\begin{array}{llll}\text { Technology in } & \text { 414ucation, } & \text { 393-416. }\end{array}$ https://doi.org/10.1080/15391523.2009.10782536 
Tapia, H., Castillo, R.F., Sarzosa, A., \& López, R. (2020). Propuesta de modelo ODEAT para el diseño de intervenciones didácticas con integración de las TIC. Revista Convergencia Educativa, 8, diciembre, 45-64. DOI: http://doi.org/10.29035/rce.8.45

Hidson, E. (2018). Challenges to Pedagogical Content Knowledge in lesson planning during curriculum transition: a multiple case study of teachers of ICT and Computing in England (Tesis doctoral). Reino Unido, Durham University. Recuperado de http://etheses.dur.ac.uk/12623/

Hofer, M., \& Harris, J. (2011). Tipos de actividades de aprendizaje en el área de Ciencias Sociales. Recuperado de https://docplayer.es/4546783-Tipos-de-actividades-de-aprendizaje-en-el-area-de-cienciassociales-1-2.html

Janssen, N., y Lazonder, A. W. (2015). Implementing Innovative Technologies Through Lesson Plans: What Kind of Support Do Teachers Prefer? Journal of Science Education and Technology, 24(6), 910-920. DOI: https://doi.org/10.1007/s10956-015-9573-5

Janssen, N., Knoef, M., \& Lazonder, A. W. (2019). Technological and pedagogical support for pre-service teachers' lesson planning. Technology, Pedagogy and Education, 28(1), 115-128. DOI: https://doi.org/10.1080/1475939x.2019.1569554

Koh, J. H. L., \& Chai, C. S. (2016). Seven design frames that teachers use when considering technological pedagogical content knowledge (TPACK). Computers y Education, (102), 244-257. DOI: https://doi.org/10.1016/j.compedu.2016.09.003

Kolb, L. (2017). Learning First, Technology Second. Portland: International Society for Technology in Education.

Kwangsawad, T. (2016). Examining EFL Pre-service Teachers' TPACK Trough Self-report, Lesson Plans and Actual Practice. Journal of Education and Learning, 10(2), 103-108. DOI: 10.11591/edulearn.v10i2.3575.

MINEDUC, \& ENLACES. (2011). Competencias y estándares TIC para la profesión docente. Santiago: MINEDUC. Recuperado de https://bibliotecadigital.mineduc.cl/handle/20.500.12365/2151

Olivares Carmona, K. M., Angulo Armenta, J., Prieto Méndez, M. E., \& Torres Gastelú, C. A. (2018). EDUCATIC: Implementación de una estrategia tecnoeducativa para la formación de la competencia digital universitaria. Píxel-Bit. Revista de Medios y Educación, (53), 27-40. DOI: https://doi.org/10.12795/pixelbit.2018.i53.02

Said Mohand, A. (2013). El diseño inverso en la adquisición de una segunda lengua: su aplicación en el desarrollo de unidades temáticas. RedELE: revista electrónica de didáctica español lengua extranjera, (25). Recuperado de https://www.educacionyfp.gob.es/dctm/redele/MaterialRedEle/Revista/2013/2013_redELE_25_05Aixa_\%20Said_Mohand.pdf?documentId=0901e72b81 61bea2 
Tapia, H., Castillo, R.F., Sarzosa, A., \& López, R. (2020). Propuesta de modelo ODEAT para el diseño de intervenciones didácticas con integración de las TIC. Revista Convergencia Educativa, 8, diciembre, 45-64. DOI: http://doi.org/10.29035/rce.8.45

Sang, G., Valcke, M., van Braak, J., \& Tondeur, J. (2010). Student teachers' thinking processes and ICT integration: Predictors of prospective teaching behaviors with educational technology. Computers y Education, 54(1), 103-112. DOI: https://doi.org/10.1016/j.compedu.2009.07.010

Schmid, M., Brianza, E., \& Petko, D. (2020). Self-reported technological pedagogical content knowledge (TPACK) of pre-service teachers in relation to digital technology use in lesson plans. Computers in Human Behavior, (115), 106586. DOI: https://doi.org/10.1016/j.chb.2020.106586

Shulman, L. S. (2005). Conocimiento y enseñanza: Fundamentos de la nueva reforma. Profesorado: Revista de currículum y formación del profesorado, 9(2), 1-30. Recuperado de https://recyt.fecyt.es/index.php/profesorado/article/view/42675

Soalablai, S. (2020). Teachers' Use of Technology in Lesson Planning and Presentation in Palau (Tesis doctoral). Walden University, Minnesota. Recuperado de https://scholarworks.waldenu.edu/cgi/viewcontent.cgi?article=10721\&context=dissertations

Tapia Silva, H. (2017). Orientaciones para la aplicación del Modelo TPACK en la formación de profesores de ERE. REER. Revista Electrónica de Educación Religiosa, 7(1), 1-24. Recuperado de http://reer.cl/index.php/reer/article/view/54

Tapia Silva, H. (2018). Actitud hacia las TIC y hacia su integración didáctica en la formación inicial docente. Actualidades Investigativas en Educación, 18(3), 1-29. DOI: https://doi.org/10.15517/aie.v18i3.34437

Tapia Silva, H., \& Sobrino Morrás, Á. (2019). Conocimiento tecnológico didáctico y del contenido de profesores chilenos. Campus Virtuales, 8(1), 121-138. Recuperado de http://rabida.uhu.es/dspace/handle/10272/16905

Vásquez Pérez, A. (2011). Plan-do-check-act en una experiencia tic en el aula: desde la idea a la evaluación. Edutec. Revista Electrónica de Tecnología Educativa, (36), a162-a162. DOI: https://doi.org/10.21556/edutec.2011.36.398

Wiggins, G., \& McTighe, J. (2005). Understanding by design. Alexandria: ASCD. Recuperado de http://library.uncg.edu/info/distance_education/wiggins.pdf 
Tapia, H., Castillo, R.F., Sarzosa, A., \& López, R. (2020). Propuesta de modelo ODEAT para el diseño de intervenciones didácticas con integración de las TIC. Revista Convergencia Educativa, 8, diciembre, 45-64. DOI: http://doi.org/10.29035/rce.8.45

\section{Datos de correspondencia}

Hugo Tapia Silva

Doctor en Educación

Universidad de La Serena, La Serena, Chile

Dirección postal: Pasaje El Morado \#681, Bosque San Carlos

Coquimbo, Chile.

ORCID ID: https://orcid.org/0000-0002-2800-034X

Email: hgtapia@userena.cl 